\title{
Anesthesia and analgesia for small animals
}

\author{
Reviewed by James Owiny, BVM, PhD, CM, DACLAM
}

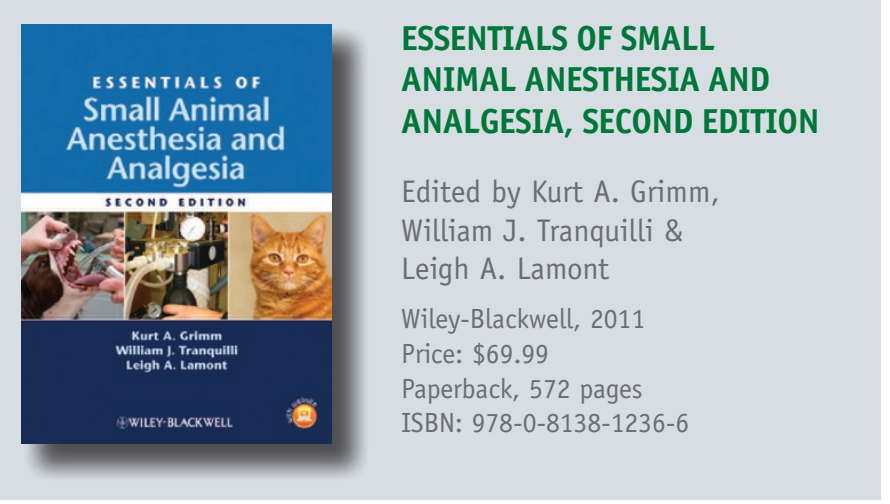

Essentials of Small Animal Anesthesia and Analgesia, Second Edition is described by the authors as "intended to provide veterinary care providers and students with the essentials of anesthetic and analgesic pharmacology, physiology, and clinical management of small animal patients." This book was published as a companion to the recently published Lumb and Jones' Veterinary Anesthesia, Fourth Edition, from which much of the content in this book originated. Forty-seven authors contributed to the original reference, and six authors have contributed new materials to this book. The authors refer readers to the original volume for more detailed information, though this reference offers a revised, updated and distilled version of the material. This book also offers new information on equipment for and management of patients with specific conditions and provides different perspectives on case management. Additional content is available from a password-protected web resource (http://www. wiley.com/go/grimm).

The book is divided into 19 chapters. The first chapter provides an introduction to patient evaluation and risk management when assessing pain management strategies. Two chapters are dedicated to providing an overview of anesthesia equipment and patient monitoring.
Pain pathophysiology and management receives a well-deserved and long overdue emphasis in this edition. Chapter 3 stresses the critical importance of prevention and control of pain in veterinary practice. Extensive definitions of all the relevant terms are provided. This is an important and often overlooked aspect in presenting materials to the audience. Nociceptive pathways and pain modulation are presented well. The authors discuss the tools used to assess acute and chronic pain nicely. A large part of this chapter is devoted to management of pain, with the primary focus being opioids and nonsteroidal anti-inflammatory agents. Acupuncture and traditional Chinese medicine is discussed in this chapter as well, an important development.

Chapters 11-19 provide examples of application of the anesthesia techniques in specific clinical situations, such as for patients with cardiovascular, respiratory, neurological, renal, liver and gastrointestinal diseases and endocrine disorders. Anesthesia for special procedures (such as ophthalmic surgery and caesarean section), for trauma cases and for critically ill patients is extensively discussed. The last chapter focuses on anesthetic emergencies and accidents.

The critical subject of euthanasia, which was covered in the first edition of Essentials of Small Animal Anesthesia and Analgesia, has unfortunately been dropped from this second edition. This subject should have been covered in this text, because it is of interest to both clinicians and veterinary students.

Although this book focuses a great deal on dogs and cats, less common species and laboratory animals are also discussed. The authors worked hard to make sure that the style is consistent among the chapters. That, together with the way the book is organized, makes it easy to follow. This consistency does result in some repetition of content in the chapters, which lengthens the book to over 500 pages. Though rather long, the book is still much shorter than the primary source and therefore a more convenient resource. The book is laid out well and easy to read and understand, making it a great and valuable resource for veterinarians, veterinary students and practitioners. 